\title{
COVID-19 literature trend and topics analyses using intelligent text mining
}

\author{
Jonathan He, Princess Anne High School, jonathanhe12345678@gmail.com \\ Rashad Saleem, Plainfield High School, rashadbsaleem@gmail.com \\ Huanmei Wu, Temple University, huanmei.wu@temple.edu \\ Jiaping Zheng, Indiana University, jizhen@iu.edu
}

\begin{abstract}
With the widespread pandemic, numerous scientific papers on COVID-19 have been published at an unprecedented rate. Keeping up with current trends and main topics of publications on the pandemic seems an impossible task. On the other hand, advanced techniques using big data analytics, machine learning, and automated text mining approaches can be used to obtain an exploratory overview of the COVID literature. This study adopts Asmussen and Møller's smart literature review framework to analyze some COVID-19 publications in the earlier period of the pandemic gathered from the COVID19 Open Research Dataset. The results show that the smart literature review framework is promising to identify hidden research themes or patterns in the published literature related to COVID- 19.
\end{abstract}

Keywords: COVID-19, text mining, literature review, topic modelling, latent Dirichlet allocation

\section{Introduction}

In response to the COVID-19 pandemic, scientific communities have published numerous academic papers at an unprecedented rate. As of June 2021, there are more than 160, 000 papers published about COVID-19 (Semantic Scholar, 2021). Over one thousand new COVID-19 related documents continue to be published every month. Multiple online portals, such as the PubMed Central (PMC) Public Health Emergency COVID-19 Initiative, the Semantic Scholar COVID-19 Open Research Dataset, and the COVID-19 Primer (Semantic Scholar, 2021; Primer, 2021; USNLM, 2021) have been set up and updated regularly as new research articles are published in conferences, journals, and other channels. Publishers such as Elsevier and Springer also provide COVID-19 paper collection for access. The rapid growth of the COVID-19 papers presents unique difficulties for researchers to keep up with research knowledge and advances in this area. It is especially difficult for researchers and healthcare practitioners to follow and keep up to date regarding the latest research findings in the fight against this pandemic.

Given the massive amounts of information in the fast-growing literature on COVID-19, a lot of effort is needed to answer questions such as:

- What can we extract from these publications to help our scientists, researchers, doctors, nurses or policymakers?

- What can we learn from what's already been published?

Systematic reviews are often used to summarize the findings in a particular area (Dickersin, Scherer, \& Lefebvre, 1994). However, systematic reviews require a lot of time and effort to complete. Given the 


\section{Issues in Information Systems}

Volume 22, Issue 4, pp. 297-304, 2021

rapid increase of the literature on COVID-19, published systematic reviews are likely to become out of date at the time of publication because they are not able to incorporate the latest articles. It is observed that some previous claims or research findings have been challenged by later articles, making many published systematic reviews less useful or of little value since they cannot stay current with the new research findings. Furthermore, the fast-growing number of papers on COVID-19 is simply too much for researchers to digest, analyze and synthesize for writing systematic reviews.

This project partially addresses the challenges posed by the fast-growing COVID-19 literature using big data analytics techniques, machine learning methods, and automated text mining approaches. The goal is to reduce the manual labor needed for extracting and analyzing the massive amounts of information, speed up the literature review, and summarize research findings on a daily, weekly, or monthly basis. In addition, automating the creation of systematic reviews by leveraging big data analytics and text mining help better usage of the COVID-19 literature, which will benefit the public, healthcare professionals, and the public.

\section{Related Work}

A literature review involves defining search keywords, searching the literature from various sources, screening the articles and synthesizing the research materials. It is estimated that conducting a systematic review takes an average of 67 weeks from registration to publication (Borah et al., 2017). To reduce researchers' workload, automating the systematic literature review is considered beneficial because it can allow researchers to better leverage their expertise and focus more on tasks that require human judgment and creativity (Pulsiri, \& Vatananan-Thesenvitz, 2018). Recently Clark et al. (2020) have completed a systematic review within two weeks by using automation tools for each of the steps of the systematic review process. There are also other types of literature reviews such as meta-analysis review, rapid review, scoping review, state-of-the-art review, conceptual review, critical Review (Grant \& Booth, 2009).

van Dinter, Tekinerdogan, and Catal (2021) did an in-depth systematic literature review to synthesize 41 primary studies that focus on the automation of Systematic Literature Review (SLR). Their review identifies the objectives of automation studies, application domains, automated steps of the SLR, automation techniques, challenges and solution directions. Technologies such as machine learning and text mining have been used to help accelerate the review process and identify relevant articles and potential areas for further research.

Marshall and Wallace (2019) provide an overview of current machine learning methods that have been proposed for research synthesis. They also introduce and compare various machine learning tools, such as RobotSearch, RCT tagger, ASReview, Thalia, RobotAnalyst, Rayyan, RobotReviewer, and SWIFTReview. The paper is helpful to expedite most steps of the systematic review process, including search, screening, and data extraction.

Text mining techniques such as latent Dirichlet allocation (LDA) and natural language processing have become a popular approach to literature analysis in recent years (Carracedo, Puertas, \& Marti, 2020). These techniques can be used to identify and extract useful insights, hidden research themes and knowledge such as relations, patterns and trends from unstructured or semi-structured data (Anderson, 2021). As a Bayesian probabilistic model for identifying latent topics from large and unstructured text documents, LDA is one of the most widely used topic modelling tools in literature analysis to group documents into the most probable matching topics (Asmussen \& Møller, 2019; Chen et al., 2020). For example, Feng et al. (2020) use LDA to analyze 62,340 papers in the field of medical informatics between 


\section{Issues in Information Systems}

Volume 22, Issue 4, pp. 297-304, 2021

1991 and 2018. Thiabaud et al. (2020) developed a Python tool to automatically extract, process, and categorize 22,709 articles and used LDA to classify and identify publications of interest, reducing the number of articles to a subset of 519 relevant articles that included 14 topics.

Asmussen and Møller (2019) developed a smart literature review framework involving three key steps: pre-processing, topic modelling, and post-processing. The pre-processing step involves getting the data and model ready to run; the topic modelling step involves the execution of the LDA method. The postprocessing step mainly translates the outcome of the LDA model to an exploratory review and using that to identify papers to be used for a detailed literature review. Their proposed smart literature review framework is shown in the following Figure 1. This framework is called smart because it supports an easy update of an exploratory review by loading newly found papers to go through the pre-processing step and re-running the LDA topic modelling without alterations to the original parameters such as number of topics.

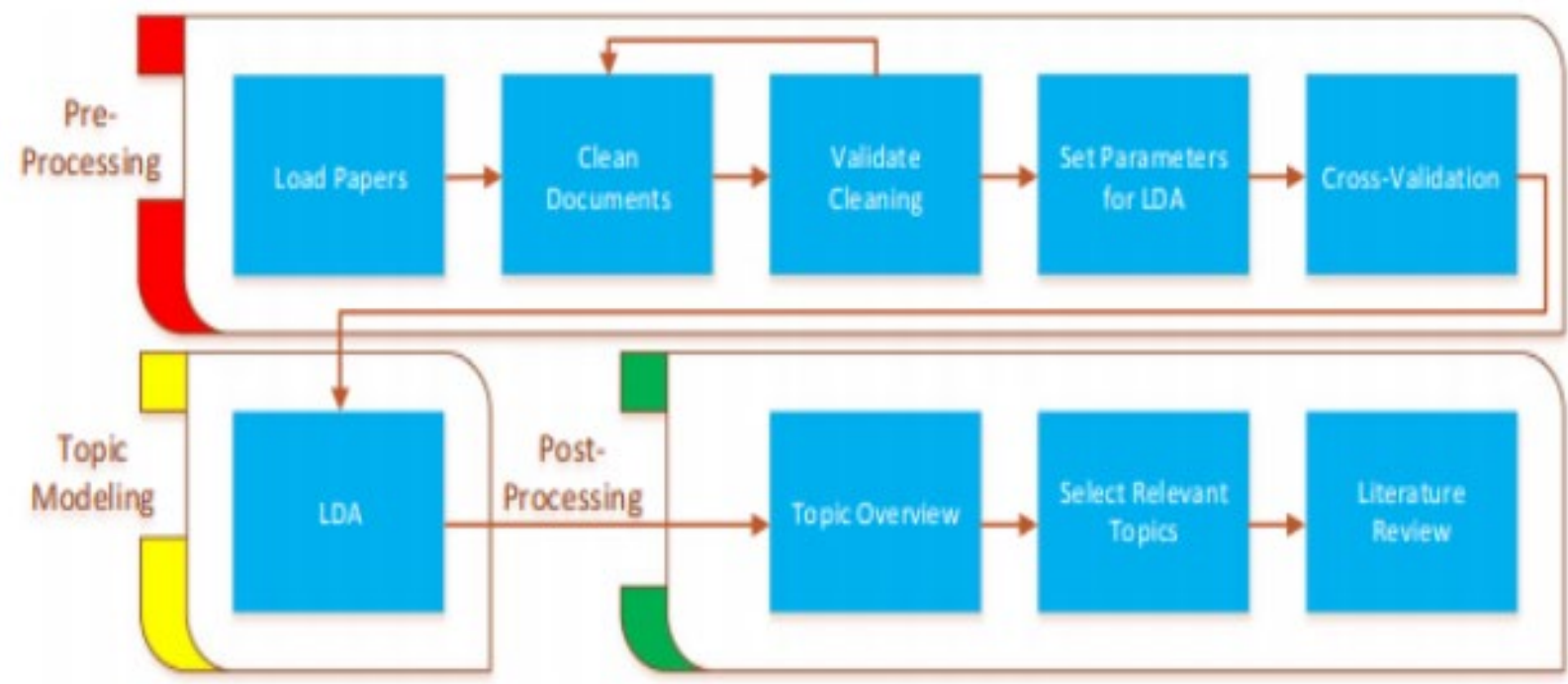

Figure 1: Asmussen and Møller (2019)'s smart literature review framework

\section{A Case Study}

Asmussen and Møller (2019)'s smart literature review framework is a suitable and convenient tool to conduct exploratory or systematic reviews on the fast-growing COVID-19 literature. To validate the framework, we did a case study by adopting their framework to analyze some earlier COVID-19 publications gathered from the COVID-19 Open Research Dataset from Semantic Scholar. It was a part of a STEM Project that provides a learning opportunity for pre-college and early college students preparing for a career in scientific research. The case study was aimed to develop a text clustering-based analysis of COVID-19 research articles. These articles were published between December 1, 2019 and June 6, 2020.

The COVID-19 corpus for our case study includes 23,000 publications. Figure 2 illustrates the detailed description of the steps for pre-processing and topic-modelling of the COVID-19 corpus. The preprocessing involved the following major steps:

- Data Cleaning: removing non-value adding words, URLs, emails, punctuation and whitespaces; all words are also converted to lowercase. 


\section{Issues in Information Systems}

Volume 22, Issue 4, pp. 297-304, 2021

- Tokenization: breaking down the text into tokens (or terms); normalizing tokens that look similar and convey the same meaning into a single canonical form (Jiang \& Zhai, 2007); approximately 27,208, 222 tokens were identified.

- Part of speech (POS) tagging: annotating syntactic categories for each word in a corpus; identifying verbs, nouns, etc.

- Stop words removal: removing common stop words such as "an", "the", "and", and "can" from standard publicly available lists (DESL, 2020).

Next, a python program was developed to extract unique keywords from full texts of these publications by using the widely used TF-IDF algorithms (Drucker, Shahrary, \& Gibbon, 2002). We inferred topic trends by applying the LDA models. The results were visualized with the PyLDAvis (an LDA visualization tool).

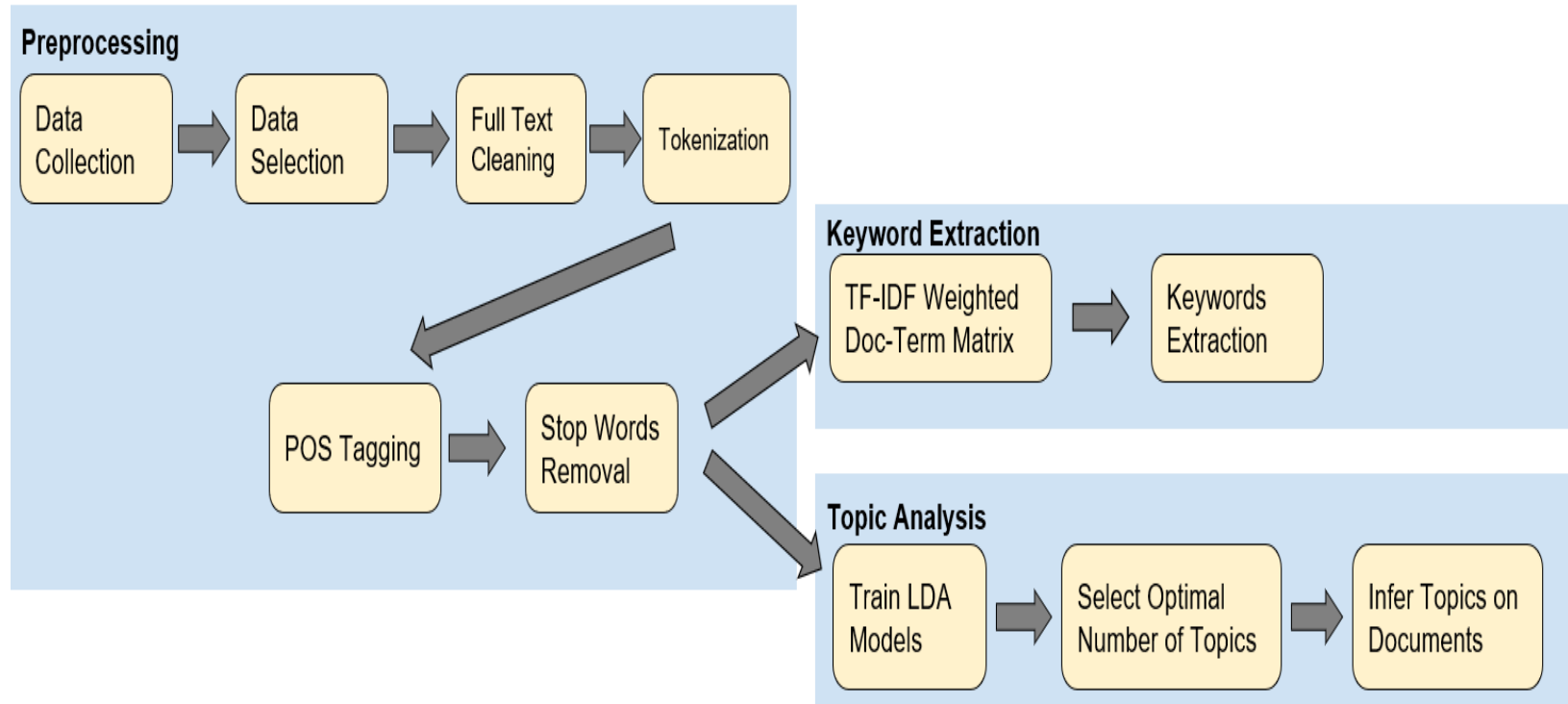

Figure 2: Our Processes and Models Used for Data Analysis

Figure 3 shows the relative frequencies of keyword categories. It is observed that keyword relative frequency groups started with big jumps. Some groups eventually maintained steady frequency, and some continued to fluctuate over time. 


\section{Relative Frequencies of Keyword Categories}

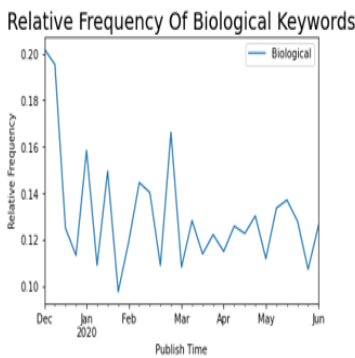

Relative Frequency Of Medical Keywords

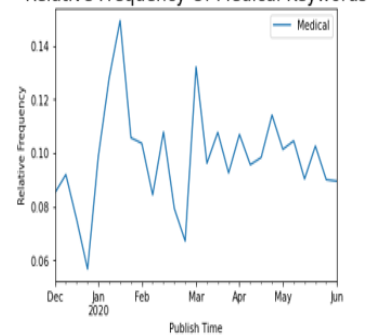

Relative Frequency Of Statistical Keywords

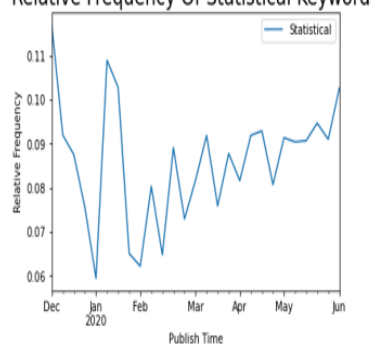

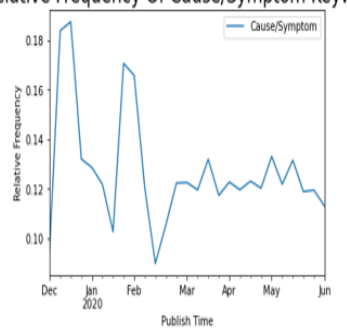
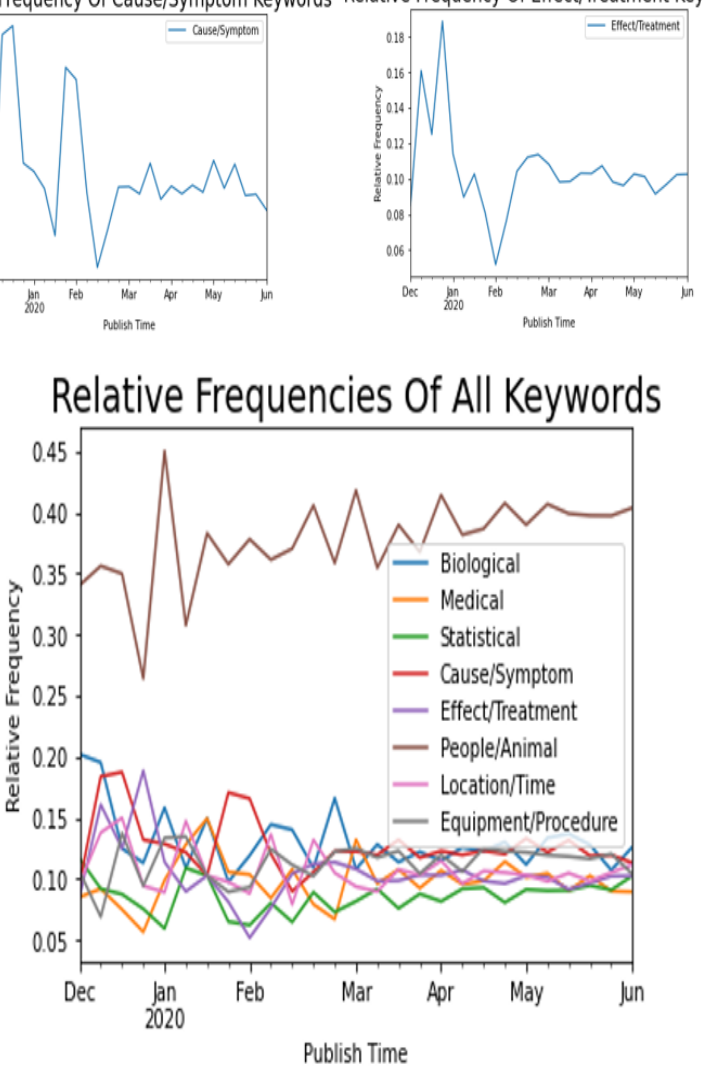

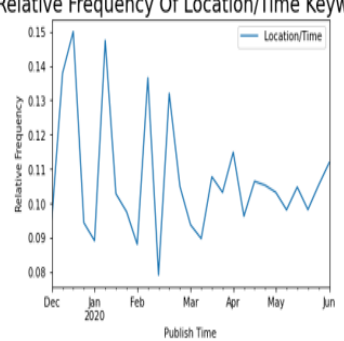

Relative Frequency Of People/Animal Keywords

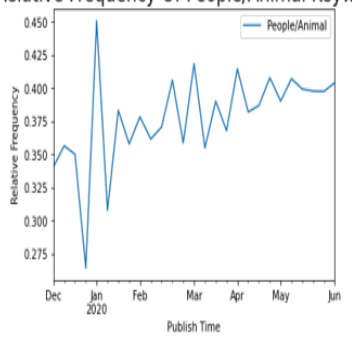

Relative Frequency Of Procedure/Equipment Keyworc

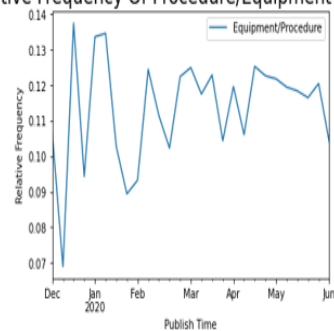

Figure 3: Relative frequencies of keyword categories

As a result of using LDA for topic modeling, we identified nine dominant topics from these publications. Figure 4 shows the visualization of a topic model and top-30 most salient words. The leading nine topics are 1) Cause/symptom, 2) Medical, 3) Statistical, 4) Epidemiology, 5) Effect/treatment, 6) People/animal, 7) Location/Time, 8) Equipment/Procedure, and 9) Biological related. Figure 5 shows the percentage of papers across the nine dominant topics (some research topics have more publications than other topics) over time, inferring topic trends during our research period. Identified topics show emphasis on treatments, respiratory problems, and transmission. 


\section{Issues in Information Systems}

Volume 22, Issue 4, pp. 297-304, 2021

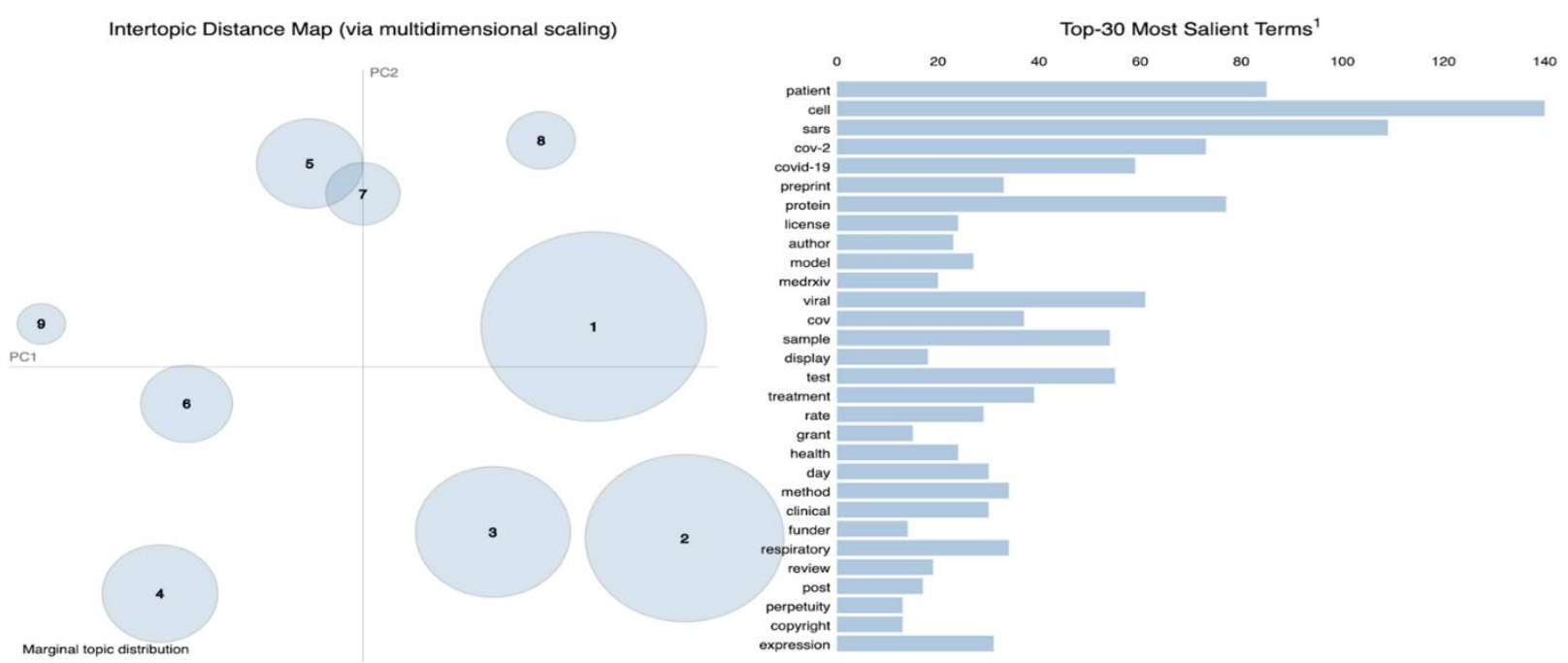

Figure 4: Visualization of the inter-topic distance map and the topic 30 Salient Terms (keywords). The left panel is the relationship and distance of the nine leading topics while right panel is the top-30 most salient terms

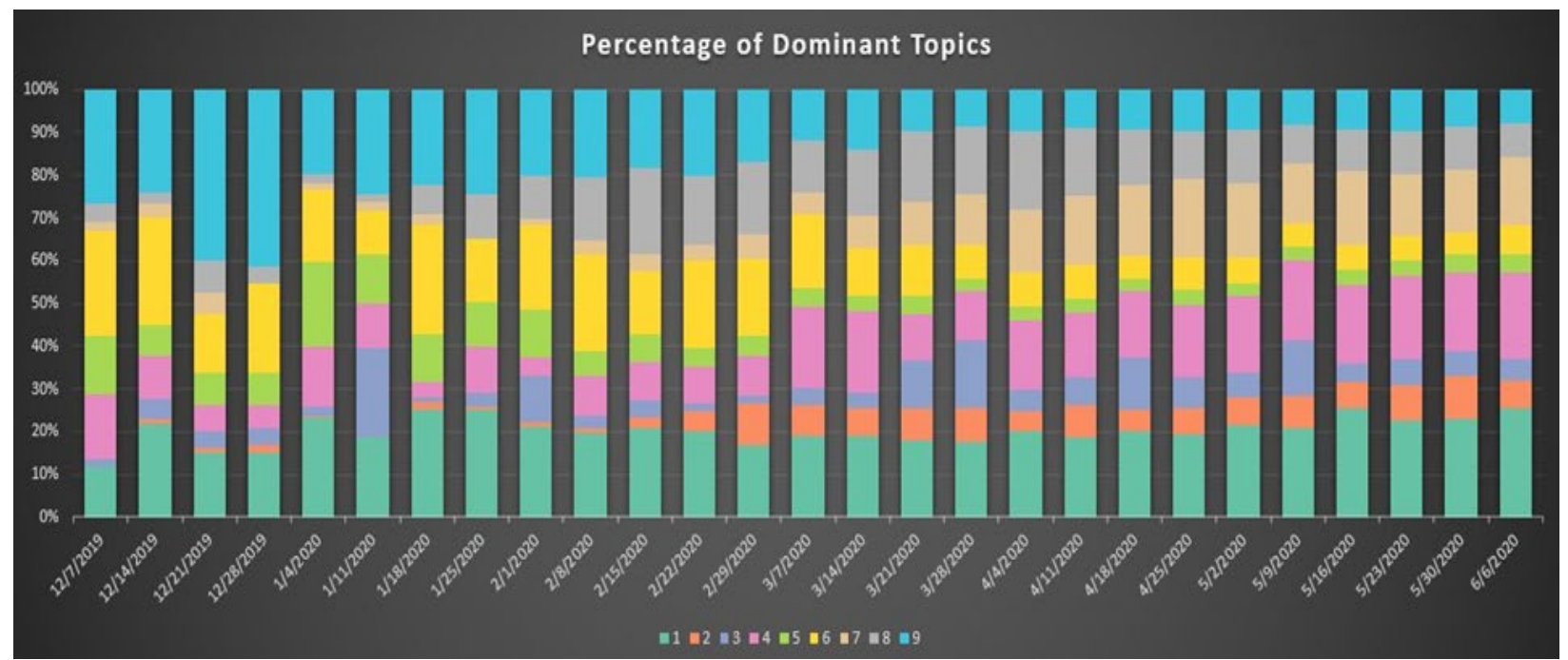

Figure 5: The percentage of papers across the nine dominant topics

The case study results suggest that Asmussen and Møller (2019)'s smart literature review framework is promising and feasible. The learning curve is not steep for researchers or students with some technical and programming capabilities. Researchers can use their framework to track the development of a research field such as COVID-19 and identify hidden research themes or patterns in the published literature related to COVID-19. Researchers can use this approach to reduce the number of articles that they need to search through, saving time to find articles relevant to their interest. By running the topic modelling script frequently for newly published papers, literature review results can be updated frequently to offer insight into emerging research themes. However, a limitation is that when the number of newly found papers is large, it is necessary to find the optimal number of topics and adjust the parameters of the topic modelling script. 


\section{Issues in Information Systems}

Volume 22, Issue 4, pp. 297-304, 2021

As for theoretical implications, this paper contributes to the literature by confirming the role of intelligent text mining, machine learning or big data techniques in facilitating the production of systematic reviews. It sheds light on developing automation methodology for systematic reviews in a timely manner and with less effort while keeping quality standards for rigorous reviews. As for practical implications, researchers may use the framework and the process introduced in this paper to narrow down the range of research articles they need to digest and gain quick insight into emerging research themes. Software developers need to continuously develop greater automation tools to help improve the speed and accuracy of systematic reviews.

There are limitations with this study. First, we only mined a small number of papers related to the COVID-19 literature in our case study. Second, we did not leverage existing machine learning tools (e.g., RobotSearch, RCT tagger, ASReview, Thalia, RobotAnalyst, Rayyan, RobotReviewer, SWIFT-Review) for systematic reviews. A comparison of these different machine learning tools for systematic reviews can be valuable.

\section{Conclusion}

With the increasing numbers of COVID-19 publications, helping researchers to find articles and information relevant to their research interest in COVID-19 is imperative. Using machine learning or big data analytics for exploratory review of the literature can help accelerate researchers' efforts in finding solutions to the problems caused by the pandemic. The aforementioned framework and techniques reveal potential areas for COVID-19 research and can make a useful contribution to understanding COVID-19 literature. The technique is also valuable to generate living systematic reviews (Thomas et al., 2017) on specific topics related to COVID-19. A future task is to implement automatic exploratory literature review based on the Asmussen and Møller (2019)'s smart literature review framework by using existing tools or creating new tools.

\section{References}

Anderson, B. S. (2021). Using text mining to glean insights from COVID-19 literature. Journal of Information Science, 01655515211001661.

Asmussen, C. B., \& Møller, C. (2019). Smart literature review: a practical topic modelling approach to exploratory literature review. Journal of Big Data, 6(1), 1-18.

Borah, R., Brown, A. W., Capers, P. L., \& Kaiser, K. A. (2017). Analysis of the time and workers needed to conduct systematic reviews of medical interventions using data from the PROSPERO registry. BMJ open, 7(2), e012545.

Carracedo, P., Puertas, R., \& Marti, L. (2020). Research lines on the impact of the COVID-19 pandemic on business. A text mining analysis. Journal of Business Research, Volume 132, pp. 586-593.

Cheng, X., Cao, Q., \& Liao, S. S. (2020). An overview of literature on COVID-19, MERS and SARS: Using text mining and latent Dirichlet allocation. Journal of Information Science, 0165551520954674 .

Clark, J., Glasziou, P., Del Mar, C., Bannach-Brown, A., Stehlik, P., \& Scott, A. M. (2020). A full systematic review was completed in 2 weeks using automation tools: a case study. Journal of clinical epidemiology, 121, 81-90. 


\section{Issues in Information Systems}

Volume 22, Issue 4, pp. 297-304, 2021

Default English Stopwords List (2020). Ranks-NL. Amsterdam, Netherlands, https://www.ranks.nl/stopwords

Dickersin, K., Scherer, R., \& Lefebvre, C. (1994). Systematic reviews: identifying relevant studies for systematic reviews. Bmj, 309(6964), 1286-1291.

Drucker, H., Shahrary, B., \& Gibbon, D. C. (2002). Support vector machines: relevance feedback and information retrieval. Information processing \& management, 38(3), 305-323.

Feng, J., Mu, X., Wang, W., \& Xu, Y. (2020). A topic analysis method based on a three-dimensional strategic diagram. Journal of Information Science, 0165551520930907.

Grant, M. J., \& Booth, A. (2009). A typology of reviews: an analysis of 14 review types and associated methodologies. Health Information \& Libraries Journal, 26(2), 91-108.

Jiang, J., \& Zhai, C. (2007). An empirical study of tokenization strategies for biomedical information retrieval. Information Retrieval, 10(4-5), 341-363.

Marshall, I. J., \& Wallace, B. C. (2019). Toward systematic review automation: a practical guide to using machine learning tools in research synthesis. Systematic reviews, 8(1), 1-10.

Primer (2021). COVID-19 Primer. https://covid19primer.com/dashboard

Pulsiri, N., \& Vatananan-Thesenvitz, R. (2018). Improving systematic literature review with automation and bibliometrics. In 2018 Portland International Conference on Management of Engineering and Technology (PICMET) (pp. 1-8). IEEE.

Semantic Scholar (2021). COVID-19 Open Research Dataset. https://www.semanticscholar.org/cord19

Thiabaud, A., Triulzi, I., Orel, E., Tal, K., \& Keiser, O. (2020). Social, Behavioral, and Cultural factors of HIV in Malawi: Semi-Automated Systematic Review. Journal of medical internet research, 22(8), e18747.

Thomas, J., Noel-Storr, A., Marshall, I., Wallace, B., McDonald, S., Mavergames, C., ... \& Pearson, L. (2017). Living systematic reviews: 2. Combining human and machine effort. Journal of clinical epidemiology, 91, 31-37.

U.S. National Library of Medicine (2021). Public Health Emergency COVID-19 Initiative. https://www.ncbi.nlm.nih.gov/pmc/about/covid-19/

van Dinter, R., Tekinerdogan, B., \& Catal, C. (2021). Automation of systematic literature reviews: A systematic literature review. Information and Software Technology, Volume 136, 106589. 